\title{
DC Test Results of the DEMO TF React\&Wind Conductor Prototype No. 2
}

\author{
Kamil Sedlak, Pierluigi Bruzzone, Boris Stepanov, Rainer Wesche, Xabier Sarasola, Davide Uglietti, Vincenzo \\ D’Auria, Christian Vorpahl, Luigi Affinito, Luigi Muzzi, Antonio della Corte and Valentina Corato
}

\begin{abstract}
In 2013, the EUROfusion organization launched the pre-conceptual design studies of the fusion DEMO tokamak. Several options of superconducting magnet design are investigated within the scope of the project. The magnets based on $\mathrm{Nb}_{3} \mathrm{Sn}$, namely the Toroidal Field (TF) and Central Solenoid (CS) coils, can be made either using the Wind\&React technology, as in ITER, or React\&Wind (RW) technology with potentially significant costsaving benefits. Within the R\&D program of the Swiss Plasma Center, the first full-size DEMO TF RW conductor prototype, RW1, was manufactured and tested in 2015-2016. The second, improved prototype, RW2, was built and tested in 2017-2018. The experimental results collected over several test campaigns performed on four assemblies of RW2 rated for $63 \mathrm{kA}$ at $12.23 \mathrm{~T}$ are presented. The current sharing temperature $T_{c s}$ of the latest RW2 assembly in DEMO operating conditions reached $7.16 \mathrm{~K}$ after 1000 electromagnetic cycles and four thermal cycles. This $\boldsymbol{T}_{c s}$ corresponds to the effective strain Eeff $=-\mathbf{0 . 2 7} \%$, significantly exceeding the design requirement of $T_{c s} \geq 6.7 \mathrm{~K}$. If $R W 2$ have been used in ITER TF coils, the $T_{c s}$ at $68 \mathrm{kA}$ and $10.9 \mathrm{~T}$ would be $7.42 \mathrm{~K}$, i.e. approximately $1 \mathrm{~K}$ higher than most of the ITER TF samples, and that with only $55 \%$ of $\mathrm{Nb}_{3} \mathrm{Sn}$ used in ITER TF conductor. The tests of RW2 proved that the cost-saving potential of the React\&Wind technology is enormous.
\end{abstract}

Index Terms-Nb33 $\mathrm{Sn}$, React\&Wind, Superconducting cable, Tokamak.

\section{INTRODUCTION}

$\mathbf{S}$ EVERAL concepts of fusion reactor to be built after ITER $\checkmark$ are being developed all around the world, many of them called DEMO. The design of DEMO tokamak prepared in Europe is coordinated by the EUROfusion consortium [1]. The work is organized within working groups, where the WPMAG working group is responsible for the magnets. Several design options are considered for the different magnet types. Swiss

This work has been carried out within the framework of the EUROfusion Consortium and has received funding from the Euratom research and training programme 2014-2018 under grant agreement No 633053. The views and opinions expressed herein do not necessarily reflect those of the European Commission. (Corresponding author: Kamil Sedlak.)

K. Sedlak, P. Bruzzone, B. Stepanov, R. Wesche, X. Sarasola, D. Uglietti, V. D’Auria are with École Polytechnique Fédérale de Lausanne (EPFL), Swiss Plasma Center (SPC), CH-5232 Villigen PSI, Switzerland (e-mail: kamil.sedlak@psi.ch).

C. Vorpahl is with EUROfusion, PMU PPPT, Boltzmann Strasse 2, D-85748 Garching, Germany.

L. Affinito, L. Muzzi, A. della Corte and V. Corato are with ENEA, 00044 Frascati, Italy.

Color versions of one or more of the figures in this paper are available online at http://ieeexplore.ieee.org.

Digital Object Identifier will be inserted here upon acceptance.
Plasma Center (SPC) of EPFL, supported by ENEA (Italy), Forschungszentrum Jülich (Germany) and other European laboratories and companies, came up with an advanced concept of the toroidal field (TF) coils based on the React\&Wind (RW) technology for the $\mathrm{Nb}_{3} \mathrm{Sn}$ conductors and on layer winding of the coil, enabling conductor grading [2].

Out of the four currently considered designs of the DEMO TF coil [3]-[6], the one proposed by SPC differs most from ITER TF. The main motivation is to reduce the overall DEMO costs by thrifty use of expensive $\mathrm{Nb}_{3} \mathrm{Sn}$ strands, to minimize the radial thickness of the TF winding pack (WP), and to simplify the coil manufacturing [7]. The quantity of required $\mathrm{Nb}_{3} \mathrm{Sn}$ is reduced by low (less negative) effective strain in the RW conductors $\left(\varepsilon_{\text {eff }} \approx-0.3 \%\right)$ compared to the Wind\&React technology employed in ITER, where $\varepsilon_{\text {eff }}$ after load cycling ranges from $-0.97 \%$ to $-0.63 \%$ [8]. The second saving effect comes from the conductor grading, where the lowestfield grade conductors contain only $25 \%$ of $\mathrm{Nb}_{3} \mathrm{Sn}$ compared to the highest-field grade [2]. The RW conductors are jacketed after heat treatment in between two longitudinally laserwelded steel half-profiles. The steel jacket can have complex shape, with different wall thickness in radial and toroidal direction, optimized for the stresses in every single layer. Grading in steel jacket reduces the radial build of the TF coil and thus the overall size of DEMO, reducing cost of other components, which is expected to be proportional to the machine volume $\left(\sim R^{3}\right)$.

\section{RW1 AND RW2 PROTOTYPES}

\section{A. Previous Measurements}

Two prototype RW TF conductors have been manufactured and tested in the past. The first one, RW1 [9], was designed for $82.4 \mathrm{kA}$ in $13.5 \mathrm{~T}$ field corresponding to the DEMO 2013 reference design. The second prototype, RW2, was optimized for $63.3 \mathrm{kA}$ and $12.2 \mathrm{~T}$ of DEMO 2015 baseline. The layout of both conductors is very similar - the $\mathrm{Nb}_{3} \mathrm{Sn}$ flat cable with thickness of only $12.3 \mathrm{~mm}$ (RW1) and $11.0 \mathrm{~mm}$ (RW2), prerequisite of low strain when cable is bent during coil winding, is surrounded by a stabilizer: a layer of thick copper wires in $\mathrm{RW} 1$, and a solid block of $\mathrm{Cu} / \mathrm{CuNi}$ "mixed matrix" stabilizer in RW2. As production of a few meters of the $\mathrm{Cu} / \mathrm{CuNi}$ stabilizer turned out to be too difficult for a laboratory but not yet 
an attractive task for a company, its procurement took more than one year.

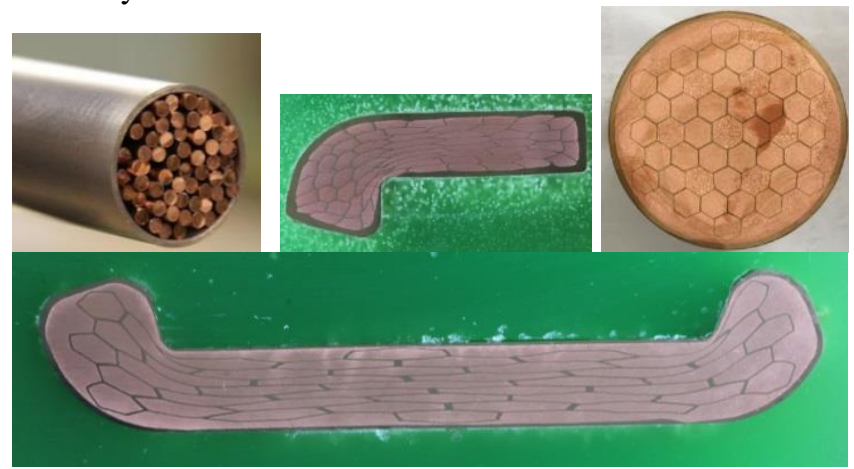

Fig. 1. Mixed matrix stabilizer: Assembly of nickel-plated copper rods inserted into CuNi can (upper left) was rolled into the half-size profile (HP, upper middle picture). In parallel, a bar extruded from a billet at WST company (upper right) was rolled to the full-size profile (FP) at Criotec.

In the meanwhile, RW2 was tested with a brass profile instead of the mixed matrix stabilizer [7]. The results of these initial tests were satisfactory, meeting the design requirement on $T_{c s}$ performance, and exhibiting very low AC loss compatible even with the typical requirements on the conductor for a central solenoid. However, a small, $\sim 0.25 \mathrm{~K}$, degradation of $T_{c s}$ during electromagnetic cycling, slightly different DC performance of two identical legs, and effective strain after cyclic loading $\varepsilon_{\text {eff }} \approx-0.43$ to $-0.45 \%$, which was below our expectation for a react\&wind conductor, called for further $R \& D$ in order to fully exploit the potential of RW technology.

Two new conductor sections were therefore assembled with some changes in the manufacturing procedure described in the following.

\section{B. Mixed Matrix Stabilizer}

Both new conductor sections were made using the mixed matrix stabilizer, replacing the temporarily used brass stabilizer used in [7]. Two manufacturing paths of the stabilizer were followed. The "half-size profiles" (HP) were produced at Criotec (Italy), under supervision of ENEA, by swaging and rolling of small $(\varnothing=30 \mathrm{~mm})$ assemblies of nickel-plated copper rods inserted into a CuNi10 can. The "full-size profiles" (FP) were produced in several steps. WST company (China) prepared a billet consisting of $55 \mathrm{CuNi}$ clad copper hexagons in a $\mathrm{CuNi}$ can. The billet was extruded and drawn down to $\emptyset=38 \mathrm{~mm}$ bars. The bars were repeatedly annealed at SPC and rolled at Criotec to the final shape of the full-size profile, see Fig. 1.

The rationale behind the mixed matrix stabilizer is to obtain a material with very good conductivity along the conductor in case of a quench, while restricting the AC loss by low conductivity in the "transverse" direction of the conductor. The latter is achieved by the layer of nickel barrier (plating or cladding) separating the copper cores.
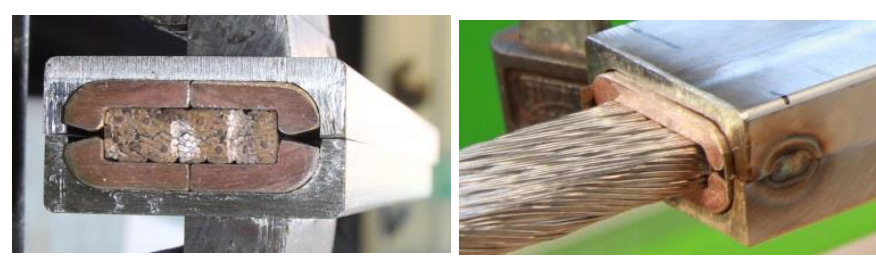

Fig. 2. Conductor assembly with the half-size profile (left) and full-size profile (right) mixed matrix stabilizer.
The RRR of both types of profiles was measured on several samples in both longitudinal and transverse direction. The results were disappointing. In the half-size profile, RRR in both longitudinal and transverse direction was very low: $\mathrm{RRR}^{\text {long }}{ }_{\mathrm{HP}}=45, \mathrm{RRR}_{\mathrm{HP}}=19$. Presumably already the copper rods used for the manufacture had low RRR (it cannot be checked, as no initial copper was left).

The RRR of the full-size profile is much higher, namely $\mathrm{RRR}_{\mathrm{FP}}^{\text {long }}=400-450, \mathrm{RRR}_{\mathrm{FP}}^{\text {trans }}=140$. The unwished high RRR in the transverse direction leads to high eddy current loss in the FP sample. The ratio of longitudinal to transverse RRR is 2.4 and 3.2 for the half-size and full-size profiles, respectively.

\section{Jacketing}

The jacket of the RW2 prototype with brass stabilizer was longitudinally laser welded at Montanstahl Company (Switzerland). The HP and FP sections were TIG welded in the SPC workshop.

During the assembly of the FP conductor, it was realized that the sum of the mixed matrix thickness $(2 \times 5.45 \mathrm{~mm})$ and cable thickness $(10.8 \mathrm{~mm})$ is $0.1 \mathrm{~mm}$ smaller than the height of the cable space within the jacket $(21.8 \mathrm{~mm})$. In operating conditions, the cable is loaded by the Lorentz force up to 12.2 $\mathrm{T} \times 63.3 \mathrm{kA}=770 \mathrm{kN} / \mathrm{m}$, and the gap between the cable and jacket further increases, leading to changes in the cable dimension (thickness), i.e. to strands movement. To avoid this, the cable was preloaded by adding an $0.25 \mathrm{~mm}$ thick steel tape interleaved on both sides between the conduit and the mixed matrix. Consequently, the allocated cable space within the jacket was $0.4 \mathrm{~mm}$ smaller than the cable thickness. The induced preload in the FP section is thus comparable to the electromagnetic load in the highest field grade layer of the DEMO TF coil.

In the assemblies of former conductor sections, with $5.35 \mathrm{~mm}$ thick brass profiles and with $5.5 \mathrm{~mm}$ thick half-size profiles, no spacer was interleaved, and the space left for the cable was $11.1 \mathrm{~mm}$ and $10.8 \mathrm{~mm}$ respectively. In all samples, a brand-new $R \& W$ cable section was used, i.e. cut from a 21 meter long RW2 cable prototype.

The average $J_{c}$ of the strands $(\varnothing=1.2 \mathrm{~mm})$ was $J_{c}(12 \mathrm{~T}, 4.2 \mathrm{~K})=1110 \mathrm{~A} / \mathrm{mm}^{2}$, normalized per non- $\mathrm{Cu}$ area.

\section{DC MEASUREMENTS}

\section{A. Current Sharing Temperature $T_{c s}$}

Two prototype RW2 conductor sections with brass profile instead of the mixed matrix stabilizer were tested in 2017 [7]. The new HP conductor section was assembled before the FP one, and it was tested together with one of the sections of the old brass-stabilizer conductor in December 2017. During this initial test phase, 200 load cycles were performed at $70 \mathrm{kA}$ and maximum achievable SULTAN field of $10.9 \mathrm{~T}$, leading to the load expected in the highest conductor grade in the DEMO operating conditions $(63.3 \mathrm{kA}$ at $12.2 \mathrm{~T})$. Afterwards, the HP section alone underwent another warm-up cool-down 
(WUCD) cycle at the beginning of July 2018 during a dedicat-

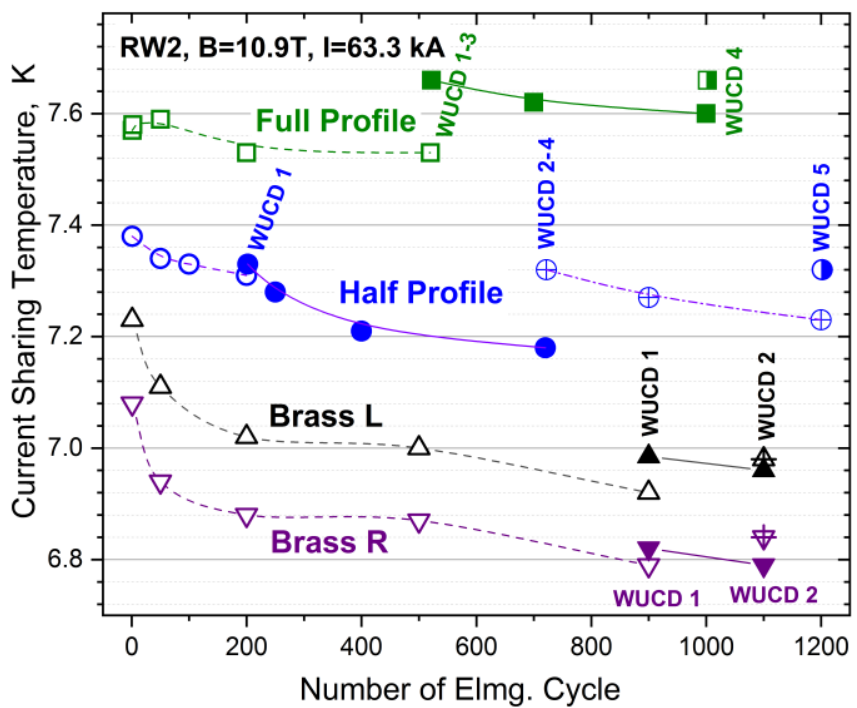

Fig. 3. Evolution of current sharing temperature $T_{c s}$ along electromagnetic cycling. Various symbol filling indicate different thermal cycles. The expected number of electromagnetic cycles in the DEMO TF coil is $\leq 1000$.

ed AC loss measurement, when HP sample was rotated by $90^{\circ}$ with respect to the usual test sample orientation. The aim was to determine AC loss along both the broad and narrow cable side of the conductor.

At the end of July 2018, a new SULTAN sample was prepared, consisting of the HP and FP conductor sections. This sample was tested during two test campaigns between July and September 2018. Four WUCD cycles were done during the testing, two planned (after 520 and 1000 load cycles), two unintentional (both after 520 load cycles) as a consequence of a cold leak in the sample.

The $T_{c s}$ along load cycling is shown in Fig. 3 . The performance of the HP conductor was better than the performance of the conductor with the brass stabilizer, however all samples show small $T_{c s}$ degradation along cycling. In the FP sample, where cable is preloaded during the jacket assembly, the $T_{c s}$ performance is clearly superior. One can even observe some

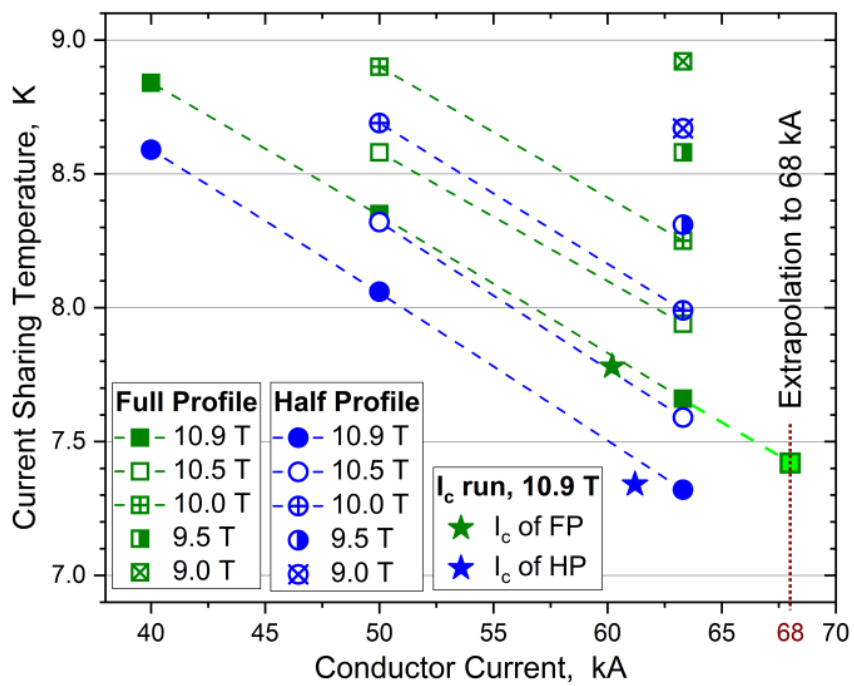

Fig. 4. Measured current sharing temperature at various currents and background magnetic fields in SULTAN. slight improvement of $T_{c s}$ during initial $\sim 50$ cycles, followed by a small drop in $T_{c s}$ of $60 \mathrm{mK}$. The performance of all samples slightly improves after every WUCD, and then slightly degrades along load cycling.

The higher $T_{c s}$ and smaller level of degradation along cyclic loading can be correlated with the preload applied on the cable during the jacket assembly. The cable in conductor sections with brass stabilizer were loose $(0.3 \mathrm{~mm}$ gap between cable with stabilizer and jacket), the HP cable was tight (no gap but also no preload), and finally FP cable was preloaded (the cable space before jacketing was $0.4 \mathrm{~mm}$ smaller than the cable thickness). This can be interpreted such that the lower freedom for strands to move during load cycling leads to a better DC performance of the flat RW conductor.

Fig. 4 shows $T_{c s}$ and $I_{c}$ measured in various operating conditions at the very end of the test campaign, i.e. after 1000 or 1200 cyclic loadings and several WUCDs. $T_{c s}$ extrapolation to ITER TF sample operating conditions in SULTAN (68 kA at $10.9 \mathrm{~T}$ ) leads to $T_{c s}=7.42 \mathrm{~K}$.

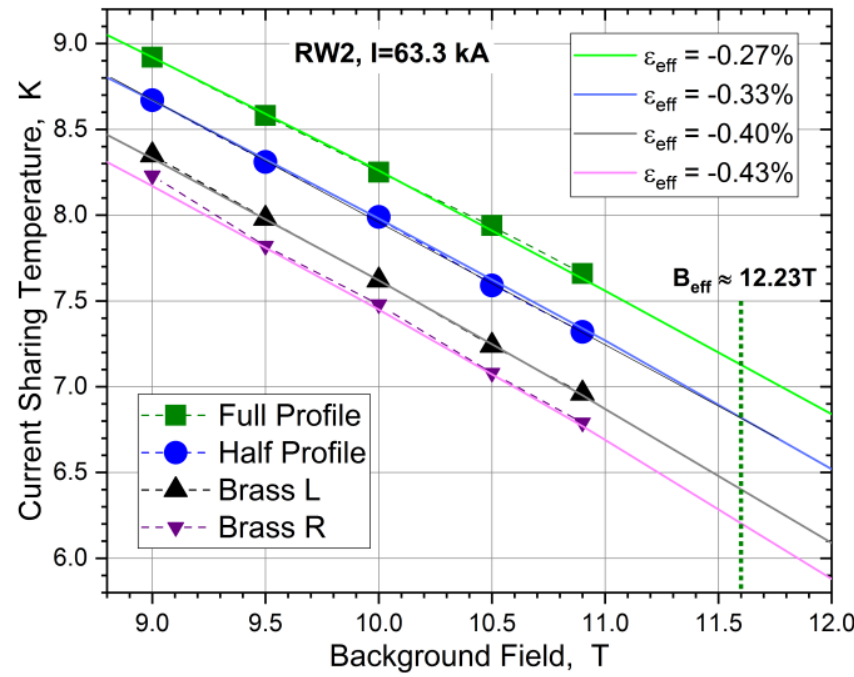

Fig. 5 Current sharing temperature $T_{c s}$ as a function of the SULTAN background field with extrapolation to the design operating field $B_{e f f}=12.23 \mathrm{~T}$.

The DEMO design operating field of the highest grade RW2 conductor is $12.23 \mathrm{~T}$. This field is beyond the range of SULTAN facility, even when taking into account the self-field and the field generated by the "return" conductor section [10], which increases the effective field $B_{\text {eff }}$ sensed by the sample by $\sim 5.4 \%$ (for $63.3 \mathrm{kA}, 10.9 \mathrm{~T}$ and given sample geometry). In order to assess $T_{c s}$ at the design field of $12.23 \mathrm{~T}$, an extrapolation of $T_{c s}$ measured at lower fields is done in Fig. 5. The extrapolated $T_{c s}$ values are $6.26 \mathrm{~K}$ and $6.43 \mathrm{~K}$ for the RW2 samples with brass stabilizer, $6.80 \mathrm{~K}$ for RW2 HP and $7.16 \mathrm{~K}$ for RW2 FP. The data can be fitted with a theoretical prediction based on single strand measurements using the effective strain $\varepsilon_{\text {eff }}=-0.43 \%$ and $\varepsilon_{\text {eff }}=-0.40 \%$ (RW2 with brass), $\varepsilon_{\text {eff }}=-$ $0.33 \%(\mathrm{RW} 2 \mathrm{HP})$ and $\varepsilon_{\text {eff }}=-0.27 \%$ (RW2 FP). The last value matches very closely the theoretically estimated average thermal strain $\varepsilon_{\text {thermal }}=-0.28 \%$ [9] for this type of RW cable. 


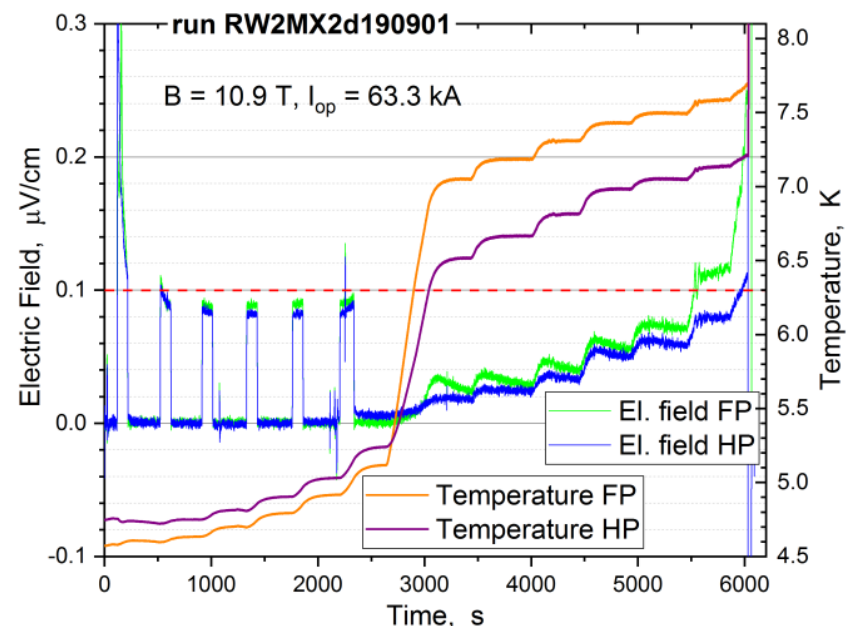

Fig. 6. An example $T_{c s}$ run taken after 1000 load cycles. The averaged and smoothed electric field barely exceeds the nominal critical electric field conventionally set to $0.1 \mu \mathrm{V} / \mathrm{cm}$ (dashed line). Current was increased in steps of 10, 20, 30, 40, 50 and $63 \mathrm{kA}$. The corresponding inductive voltage induced during the current ramp-ups is visible during the initial $2300 \mathrm{~s}$.

\section{B. Take-off Voltage}

A typical $T_{c s}$ run is shown in Fig. 6. The voltage developed in the $45 \mathrm{~cm}$ long high-field SULTAN region is measured by six pairs of voltage taps in each conductor section. The electric field shown in Fig. 6 is calculated from the average voltage signal divided by $45 \mathrm{~cm}$, corrected for a small dependence of voltage on current [11], and smoothed. As can be seen, the take-off voltage only slightly exceeds the critical electric field conventionally set to $0.1 \mu \mathrm{V} / \mathrm{cm}$. Unlike in the degraded ITER TF conductors, where the take-off voltage is several times higher (see e.g. Fig. 3 of [12]), and increases after cyclic loading, the take-off voltage in RW2 conductor remained very low (slightly above $0.1 \mu \mathrm{V} / \mathrm{cm}$ ) during the whole test campaign. The nominal operating current density per strand area in RW2 cable is $239 \mathrm{~A} / \mathrm{mm}^{2}$, to be compared with $143 \mathrm{~A} / \mathrm{mm}^{2}$ in the ITER TF cables. The low take-off voltage resembles the behavior of NbTi conductors.

\section{The n-value}

Several $I_{c}$ runs were taken during the test campaigns. The measured $I_{c}$ corresponds to the measured $T_{c s}$, being slightly below the value expected from the $T_{c s}$ measurements, as shown in Fig. 4. Due to the low take-off voltage, the n-index was determined from a fit in relatively low electric field region, close to $0.1 \mu \mathrm{V} / \mathrm{cm}$. The initial $\mathrm{n}$-value in FP section was 20 , and it was gradually increasing during the test campaign to the final value of 25 after the last WUCD. In the HP section the n-value was close to 20 in most of the runs, except two intermediate runs when it dropped to 16 . The relatively high $\mathrm{n}$ value at the end of the test campaign suggests that there was no significant irreversible degradation (filament breakage) during the test campaign. (The $n$-value in ITER TF samples after cyclic loading is often around 10, see e.g. Fig. 11 in [8].)

\section{CONCLUSION}

The testing of react\&wind sample RW2 was done in several test campaigns on four different conductor sections. The DC performance of the sample improved once the cable movement was restricted by the tight fit into the cable space, and it got even better when the cable was preloaded. The current sharing temperature $T_{c s}$ of the latest RW2 prototype in DEMO operating conditions reached $7.16 \mathrm{~K}$ after 1000 electromagnetic cycles and four WUCD cycles, providing a temperature margin higher than the required $1.5 \mathrm{~K}$. The effective strain in the preloaded sample at the end of the test campaign is $\varepsilon_{\text {eff }}=-0.27 \%$, i.e. very close to the expected thermal strain $\left(\varepsilon_{\text {thermal }}=-0.28 \%\right)$, and the degradation along cyclic loading was insignificant.

The $\mathrm{Nb}_{3} \mathrm{Sn}$ cross-section in the RW2 FP conductor is $132 \mathrm{~mm}^{2} . T_{c s}$ at $68 \mathrm{kA}$ and 10.9 T SULTAN background field is $T_{c s}=7.42 \mathrm{~K}$. This number can be compared to $T_{c s}$ of ITER TF conductors at the same current and field, which is in the range $6.3 \mathrm{~K}-6.5 \mathrm{~K}$ (for conductors made of internal-tin strands, [8]), where $238 \mathrm{~mm}^{2}$ of $\mathrm{Nb}_{3} \mathrm{Sn}$ cross-section is used, i.e. $80 \%$ more compared to RW2. This, together with the additional $\mathrm{Nb}_{3} \mathrm{Sn}$ saving due to conductor grading, promises significant cost reduction of DEMO TF magnets.

The low take-off voltage and high n-index resemble behavior of NbTi conductors, probably as a consequence of approaching the operating conditions close to the theoretical maximum of $J_{c}$ for a given current and magnetic field at zero strain.

The measurements of AC loss will continue with a dedicated sample made of single conductor section rotated by $90^{\circ}$ and will be described and discussed in a separate paper. The AC measurements performed so far indicate that the coupling loss in the RW2 cable is satisfactorily low, while eddy current loss in the full-size mixed-matrix stabilizer dominate the overall AC loss, and further R\&D is needed to reduce it. This could be done either by improving the internal structure of the mixed matrix, or by replacing it with highly-compacted claddedcopper Rutherford cables.

\section{ACKNOWLEDGMENT}

The authors would like to thank the WST for donating the $\mathrm{Nb}_{3}$ Sn strand used for the production of RW2 cable, TRATOS for the cabling work, and WST and Criotec for the mixed matrix production. The authors also thank Paul Scherrer Institute for its technical support, and M. Husistein, F. Roth, F. Oberle, A. Kreuzer, R. Smith, M. Jeni, C. Müller und M. Holenstein for their careful and dedicated sample preparation and enthusiastic SULTAN operation. We also commemorate our dear colleague Anatoly Panin, who valuably contributed to the mechanical design of the RW2 conductor, and who passed away in March 2018. 


\section{REFERENCES}

[1] G. Federici et al., "Overview of the design approach and prioritization of R\&D activities towards an EU DEMO," Fusion Eng. Des. 109-111, 2016, pp. 1464-1474.

[2] K. Sedlak, P. Bruzzone, X. Sarasola, B. Stepanov and R. Wesche, "Design and R\&D for the DEMO Toroidal Field Coils Based on $\mathrm{Nb}_{3} \mathrm{Sn}$ React and Wind Method," IEEE Trans. Appl. Supercond., vol. 27, no. 4, 2017, Art. no. 4800105.

[3] L. Muzzi et al., "Design, Manufacture, and Test of an $80 \mathrm{kA}$-Class $\mathrm{Nb}_{3} \mathrm{Sn}$ Cable-In-Conduit Conductor With Rectangular Geometry and Distributed Pressure Relief Channels," IEEE Trans. Appl. Supercond., vol. 27, no. 4, 2017, Art. no. 4800206.

[4] M. B. Biancolini, R. Bonifetto , A. Chiappa , F. Giorgetti , V. Corato, L. Muzzi and S. Turtu, "Mechanical Analysis of the ENEA TF Coil Proposal for the EU DEMO Fusion Reactor," IEEE Trans. Appl. Supercond., vol. 28, no. 4, 2018, Art. no. 4901405.

[5] A. Torre, D. Ciazynski and L. Zani, "EU-DEMO TF and CS Magnet Systems Design and Analyses Performed at CEA," IEEE Trans. Appl. Supercond., vol. 27, no. 4, 2017, Art. no. 4900705.

[6] R. Heller, P. V. Gade, W.H. Fietz, T. Vogel and K.-P.Weiss, "Conceptual Design Improvement of a Toroidal Field Coil for EU DEMO Using High-Temperature Superconductors," IEEE Trans. Appl. Supercond., vol. 26, no. 4, 2016, Art. no. 4201105.

[7] P. Bruzzone et al., "A Prototype Conductor by React\&WIND Method for the EUROfusion DEMO TF Coils," IEEE Trans. Appl. Supercond., vol. 28, no. 3, 2018, Art. no. 4202705.

[8] M. Breschi, D. Macioce and A. Devred, "Performance analysis of the toroidal field ITER production conductors," Supercond. Sci. Technol. 30, 2017, Art. no. 055007.

[9] P. Bruzzone, K. Sedlak, B. Stepanov, R. Wesche, L. Muzzi, M. Seri, L. Zani and M. Coleman, "Design, Manufacture and Test of a $82 \mathrm{kA}$ React\&Wind TF Conductor for DEMO," IEEE Trans. Appl. Supercond., vol. 26, no. 4, 2016, Art. no. 4801805.

[10] R. Wesche, B. Stepanov, A. Anghel, S. Huber, G. Pasztor, M. Vogel and P. Bruzzone, "Self-field effects in NbTi subsize cable-in-conduit conductors," Physica C, 401, pp. 113-117, 2004.

[11] P. Bruzzone, B. Stepanov, R. Wesche, Y. Ilyin, R. Herzog, M. Calvi, M. Bagnasco and F. Cau, "Methods, Accuracy and Reliability of ITER Conductor Tests in SULTAN," IEEE Trans. Appl. Supercond., vol. 19, no. 3, 2009, pp. 1508-1511.

[12] M. Breschi et al., "Results of the TF conductor performance qualification samples for the ITER project," Supercond. Sci. Technol. 25, 2012, Art. no. 095004. 